\title{
Implementation of primary HPV testing in Japan
}

\author{
TETSUJI KUROKAWA ${ }^{1}$, YOSHIO YOSHIDA ${ }^{1}$, OSAMU IWANARI ${ }^{2}$, TETSURO OISHI $^{3}$, \\ TOKUZO KASAI ${ }^{4}$, MASAO HAMADA ${ }^{5}$, HIROMASA FUJITA ${ }^{6}$, HIROYUKI FUJIWARA ${ }^{7}$, \\ MASATOSHI YOKOYAMA ${ }^{8}$, NORIAKI SAKURAGI ${ }^{9}$, JUNZO KIGAWA $^{10}$ and MITSUAKI SUZUKI ${ }^{11}$ \\ ${ }^{1}$ Department of Obstetrics and Gynecology, Faculty of Medical Sciences, University of Fukui, Fukui 910-1193; \\ ${ }^{2}$ Department of Obstetrics and Gynecology, Shimane Prefectural Central Hospital, Izumo, Shimane 693-8555; \\ ${ }^{3}$ Department of Obstetrics and Gynecology, Tottori University, Yonago, Tottori 683-8504; ${ }^{4}$ Chiba Foundation for Health \\ Promotion and Disease Prevention, Chiba 261-0002; ${ }^{5}$ Hamada Ladies Clinic, Miyazaki 880-0121; \\ ${ }^{6}$ Hokkaido Cancer Society, Sapporo, Hokkaido 065-0026; ${ }^{7}$ Department of Obstetrics and Gynecology, Jichi Medical \\ University, Shimotsuke, Tochigi 329-0498; ${ }^{8}$ Department of Obstetrics and Gynecology, University of Saga, Saga 849-8501; \\ ${ }^{9}$ Department of Obstetrics and Gynecology, Otaru General Hospital, Otaru, Hokkaido 047-8550; \\ ${ }^{10}$ Department of Obstetrics and Gynecology, Matsue City Hospital, Matsue, Shimane 690-8509; ${ }^{11}$ Department \\ of Obstetrics and Gynecology, Shinyurigaoka General Hospital, Kawasaki, Kanagawa 215-0026, Japan
}

Received November 17, 2019; Accepted June 17, 2020

DOI: $10.3892 / \mathrm{mco} .2020 .2092$

\begin{abstract}
Cervical cancer screening has been shifting from primary cytology to primary HPV testing worldwide as primary HPV testing is more sensitive than primary cytology. To the best of our knowledge, the current study is the first in Japan to examine the feasibility of primary HPV testing. One of the disadvantages of this shift is that hrHPV-/ $\geqq$ LSIL/CIN2+ (high-risk HPV negative cancers or pre-cancerous lesions with abnormal cytology results) can be missed. The objectives of the present study are to clarify in detail CIN2+ missed by this shift and to evaluate the feasibility of primary HPV testing in Japan. Data from 115,273 women who underwent co-testing with cytology and HPV testing in cancer screening were used in the current study. The cases with $h r H P V-/ \geq L S I L$ (' $h r H P V-/ \geq L-S I L '$ include CIN2-, in contrast, ' $h r H P V-/ \geqq L-S I L / C I N 2+'$ doesn't include CIN2-) were analysed in detail. Women with hrHPV-/ $\geqq$ LSIL comprised $0.3 \%$ of the total. The prevalence of CIN2, CIN3, SCC or cervical adenocarcinomas in the lesions with HPV-/ $\geqq$ LSIL was $0.03 \%$ in the cancer screening group. Only one case of 14 cervical adenocarcinomas in $\geqq$ LSIL was hrHPV-. The prevalence of cancer missed by the shift in patients $>50$ years of age was significantly higher compared with patients younger than 49 years. In conclusion, the preva-
\end{abstract}

Correspondence to: Dr Tetsuji Kurokawa, Department of Obstetrics and Gynecology, Faculty of Medical Sciences, University of Fukui, 23-3 Matsuoka, Shimoaizuki, Eiheiji-cho, Yoshida-gun, Fukui 910-1193, Japan

E-mail: kurotetu@u-fukui.ac.jp

Key words: uterine cervical cancer, cancer screening, cytology, primary HPV testing, HPV negative cancers lence of CIN2+, which might be missed by the shift from primary cytology to primary HPV testing, was remarkably low in this Japanese cancer screening. The data indicated that primary HPV testing, which was more sensitive for CIN2+ than primary cytology, was a feasible method that can be used in Japan. In particular, primary HPV testing should be introduced for women $<50$ years old.

\section{Introduction}

In 2018, uterine cervical cancer ranked third in both estimated new cases and deaths in females in the world (1). The estimated age-standardized incidence rate in Eastern Asia, including Japan, is higher than that in North America (2). The high incidence rate of cervical cancer is a crucial public health problem for women in Japan. The oncogenic mechanisms of cervical cancer are well known. Almost all cervical cancers are associated with a persistent infection with human papillomavirus (HPV). Therefore, the WHO states that actions such as vaccination against HPV, screening and treatment of pre-cancer, early detection and prompt treatment of early invasive cancers and palliative care must be embedded in health systems aimed at delivering universal health coverage and that now is the time for global elimination. (https://www.who.int/cancer/cervical-cancer) The preventive actions of this strategy are two-fold. One is the vaccination against HPV infection as a primary prevention. The other is cancer screening for early detection as secondary prevention. Unfortunately, in Japan, the HPV vaccination rate is only $1 \%$ because the Japanese Ministry of Health, Labor and Welfare has suspended proactive recommendations for the HPV vaccination programme (3). Thus, to prevent cervical cancer in Japanese women, cancer screening has played an important role.

Three programmes for cervical cancer screening have been recommended worldwide: Primary cytology, co-testing 
with cytology and HPV test (co-testing), and primary HPV testing (4). Cancer screening has shifted from primary cytology to co-testing or primary HPV testing (5). The cancer screening environment is different in different countries. Each country has chosen the programme they feel is optimal by verifying the balance between benefit and harm in each program (5-12). In Japan, hardly any areas have introduced primary HPV testing. Therefore, we must analyse hrHPV-/CIN2+/ミLSIL (high-risk HPV negative cancerous or pre-cancerous lesions with abnormal cytology results) in detail, which are missed by primary HPV testing without cytology. The objective of the present study is to clarify the prevalence and histology of hrHPV-/CIN2+/ミLSIL from a Japanese cancer screening programme and to evaluate the feasibility of primary HPV testing in Japan. This report is the first to study hrHPV-/CIN2+/ミLSIL using big data from 115,273 women who underwent cancer screening.

\section{Materials and methods}

Population. Data from 115,273 women who underwent cervical cancer screening in eight areas (Hokkaido 16,322; Tochigi 21,338; Chiba 17,292; Fukui 7,573; Tottori 19,664; Saga 3,064; Miyazaki 19,858; Shimane 10,162) in Japan were used for this study. The data of Hokkaido were from 2013. The data of Tochigi and Chiba were from 2012-2014. The data of Fukui were from 2015. The data of Tottori were from 2013-2017. The data of Saga were from 2013. The data of Miyazaki and Shimane were from 2014-2016. We obtained consent from all participants using the opt-out system. All women underwent both cytology and HPV testing. All women were older than 20 years of age. This study was approved by the Ethics Review Board of Fukui University (approval no. 20190094).

Cytology. In cytological samples, $25.6 \%$ were analysed using conventional cytology, and $74.4 \%$ were analysed using liquid-based cytology. The cytologists were blinded to the HPV results and reported their results using the Bethesda 2001 System (13). In this study, LSIL (low-grade squamous intraepithelial lesion) or worse, which indicated the need to undergo colposcopy, were defined as abnormal cytology results.

HPV testing. In the HPV test, 59.6\% used Hybrid Capture 2 (HC2; Qiagen Inc.), and 40.4\% used Cobas HPV (Roche). HC2 can detect 13 different oncogenic HPV genotypes: 16, 18, 31, 33, 35, 39, 45, 51, 52, 56, 58, 59 and 68 (14). Cobas HPV can be detected in three separate channels: HPV16 individually, HPV18 individually, and a pool of 12 other HPV genotypes: 31, 33, 35, $39,45,51,52,56,58,59,66$ and 68 (14). Negative results for the HC2 test or Cobas HPV test were treated as hrHPV-(negative high-risk HPV) in this study.

Data analysis. The data collected by co-testing were simulated as a model for the primary HPV test. The confidence interval was calculated at $95 \%$. The difference in the prevalence of cancer with hrHPV-/ $\geqq$ LSIL between less than 49 and more than 50 was analysed using the Fisher's test. In the discussion, the difference between other studies and our study was also analysed using the Fisher's test. P-values $<0.01$ were consid-
Table I. The total numbers by age group in 8 regions.

\begin{tabular}{lrc}
\hline Age group & Number & Rate \% $(95 \%$ CI $)$ \\
\hline $20-24$ & 2,823 & 2.4 \\
$25-29$ & 9,121 & 7.9 \\
$30-34$ & 14,216 & 12.3 \\
$35-39$ & 14,799 & 12.8 \\
$40-44$ & 17,473 & 15.2 \\
$45-49$ & 12,949 & 11.2 \\
$50-54$ & 7,501 & 6.5 \\
$55-59$ & 8,189 & 7.1 \\
$60-$ & 28,202 & 24.5 \\
Total & 115,273 & 100 \\
\hline
\end{tabular}

CI, confidence interval.

ered to indicate a statistically significant difference. We used Bell Curve for Excel (v3.20) for all analyses.

\section{Results}

Results by age group in 8 regions. We analysed the 115,273 women who underwent cervical cancer screening by the government programme in 8 regions. The highest rate was $24.5 \%$, which was observed in women older than 60 years. The lowest rate was $2.4 \%$ in women $20-24$ years old. Overall, a mountain-shaped curve was observed, with a peak in women 40-44 years old. There was no significant difference in the population ratio among age groups (Table I).

The distribution of cytology results by HPV status. We found that the percentage of women with hrHPV+ (positive high-risk HPV) was 7.4\%, whereas the percentage of women with hrHPV- was $92.6 \%$. Table II shows the women with hrHPV-/ $\geqq$ LSIL were found in only $0.3 \%$. Moreover, in the women with $\geqq \mathrm{LSIL}$, the prevalence of hrHPV- was $12.0 \%$ $(298 / 2,491)$. Table II indicates that the number of women with hrHPV-/ $\geqq$ LSIL who were missed by the primary HPV test was extremely low (Table II).

The distribution of pathological results in women with $h r H P V-/ \geqq L S I L$. In 298 cases, 255 cases could be analysed and 43 cases could be not analysed (36 cases of no biopsy and seven cases of inadequate biopsy). Approximately half of the women with hrHPV-/ $\geqq$ LSIL had no lesions according to the pathological results. No lesions or CIN1, which did not require treatment or additional examination, were found in $81.17 \%(207 / 255)$ of women. CIN2, CIN3 or SCC were found in $14.90 \%(38 / 255)$. Cervical adenocarcinoma was found in $0.39 \%(1 / 255)$. Only one case of 14 cervical adenocarcinomas with $\geqq$ LSIL was hrHPV-. Nine cases of endometrial adenocarcinomas were found. Although we were concerned regarding the difficulty of detecting cervical adenocarcinoma by primary HPV test, our results suggested that the frequency of cervical adenocarcinoma missed by the primary HPV test was much lower than expected (Table III). 
Table II. The distribution of cytology results by HPV status.

\begin{tabular}{|c|c|c|c|c|}
\hline \multirow[b]{2}{*}{ Cytology result } & \multicolumn{2}{|c|}{ HPV-positive } & \multicolumn{2}{|c|}{ HPV-negative } \\
\hline & Number & Rate $\%(95 \%$ CI) & Number & Rate \% (95\% CI) \\
\hline NILM & 5,232 & $4.54(4.42-4.66)$ & 105,590 & $91.60(91.44-91.76)$ \\
\hline ASC-US & 1,085 & $0.94(0.89-1.00)$ & 875 & $0.76(0.71-0.81)$ \\
\hline$\geqq \mathrm{L}-\mathrm{SI}$ & 2,193 & $1.90(1.82-1.98)$ & 298 & $0.26(0.23-0.29)$ \\
\hline Total & 8,510 & $7.38(7.23-7.53)$ & 106,763 & $92.62(92.47-92.77)$ \\
\hline
\end{tabular}

CI, confidence interval; HPV, human papillomavirus; NILM, negative for intraepithelial lesion or malignancy; ASC-US, atypical squamous cells of undermined significance; L-SIL, low grade-squamous intraepithelial lesion.

Table III. The distribution of pathological results in the women with hrHPV-/æL-SIL.

\begin{tabular}{|c|c|c|}
\hline Feature & hrHPV-/ミL-SIL & $\begin{array}{l}\text { Rate }(\%) \\
(95 \% \mathrm{CI})\end{array}$ \\
\hline No lesion & 127 & $\begin{array}{c}49.80 \\
(43.71-55.90)\end{array}$ \\
\hline $\mathrm{CIN}^{\mathrm{a}}$ & 80 & $\begin{array}{c}31.37 \\
(25.71-37.04)\end{array}$ \\
\hline $\mathrm{CIN} 2^{\mathrm{b}}$ & 21 & $\begin{array}{c}8.24 \\
(4.77-11.70)\end{array}$ \\
\hline $\mathrm{CIN}^{\mathrm{c}}$ & 16 & $\begin{array}{c}6.27 \\
(3.18-9.37)\end{array}$ \\
\hline $\mathrm{SCC}$ & 1 & $\begin{array}{c}0.39 \\
(-0.89-1.68)\end{array}$ \\
\hline $\begin{array}{l}\text { Cervical } \\
\text { adenocarcinoma }\end{array}$ & 1 & $\begin{array}{c}0.39 \\
(-0.89-1.68)\end{array}$ \\
\hline $\begin{array}{l}\text { Endometrial } \\
\text { adenocarcinoma }\end{array}$ & 9 & $\begin{array}{c}3.53 \\
(1.08-5.98)\end{array}$ \\
\hline Total & 255 & 100.0 \\
\hline
\end{tabular}

CI, confidence interval; CIN1 ${ }^{\text {a }}$, cervical intraepithelial neoplasia, Grade 1; CIN2 ${ }^{\mathrm{b}}$, cervical intraepithelial neoplasia, Grade 2; CIN $3^{\mathrm{c}}$, cervical intraepithelial neoplasia, Grade 3; SCC, squamous cell carcinoma; L-SIL, low grade-squamous intraepithelial lesion.

The prevalence of carcinomas by age group in women with $h r H P V-/ \geqq L-S I L$. Table IV reveals that the prevalence of SCC, cervical adenocarcinoma or endometrial carcinoma in the women with hrHPV-/ $\geqq$ LSIL was $0.0095 \%(11 / 115,273)$ in this study. 9 women had hrHPV-/æLSIL carcinomas in women 50 years or older and the remaining 2 women had hrHPV-/ $\geqq$ LSIL carcinomas in women younger than 49 years old. The detection rate for carcinomas in women older than 50 years old $(0.0205 \%$ : $9 / 43,892)$ was significantly higher than that in women younger than 49 years old $(0.0028 \%$ : $2 / 71,381$; $\mathrm{P}<0.01)$. However, the reason of the difference is to discover endometrial carcinomas which is advantage as unexpected for cervical cancer screening. $72.7 \%$ (8/11) of cancers detected by primary cytology in Japanese cervical cancer screening were endometrial adenocarcinomas of women older than 50 years old (Table IV).

\section{Discussion}

Cervical cancer screening has shifted from primary cytology to primary HPV testing in the world (5). In Japan, the Cervical Cancer Screening Program organized by almost all municipalities currently requires primary cytology screening every 2 years for all women over 20 years old (15). The disadvantage of cytology is its low sensitivity for CIN2+. From our data, the sensitivity of this method is $70 \%$ (16). No municipalities in Japan have introduced the primary HPV testing because of a lack of Japanese data. This is the first study in Japan to examine the feasibility of the primary HPV testing. Studies on hrHPV-/CIN2+, which cannot be detected by the primary HPV test, are essential to introduce primary HPV testing in Japan. In the Belgian cancer register, the prevalence in hrHPV negative cancers was $14.7 \%$ (17). In some literatures, the percentage of hrHPV negative cancers ranges from 7 to $11 \%$ (17-19). A previous report from Japan suggested that $1.5 \%$ of cervical cancers and $3.8 \%$ of CIN2-3 were hrHPV- (20). The prevalence range was wide because the population of each study varied. From this prevalence, we cannot determine whether primary HPV testing is an optimal strategy in Japan. We focused on hrHPV-/ $\geqq \mathrm{LSIL}$ /CIN2+, which can be detected by primary cytology and missed by primary HPV testing. We conducted the present study to clarify hrHPV-/ $\geqq$ LSIL/CIN2+ in detail and then evaluate the feasibility of the primary HPV testing in Japan.

In this study, the prevalence of hrHPV-/ $\geqq$ LSIL was $0.26 \%$ in the total age groups. We compared the prevalence of hrHPV-/ $\geqq$ LSIL between the ARTISTIC study, which was a large study from the U.K. in the cancer screening population, and our study (20-24). The prevalence in our study $(0.26 \%$ : $298 / 115,273)$ was significantly lower $(\mathrm{P}<0.01)$ than that in the ARTISTIC study (1.14\%: 209/18386) (23). However, we must consider the possibility that the difference in prevalence was due to age differences (ARTISTIC 20-64 years old vs. Our study 20+ years) (23). Our study was also compared with the ATHENA study, which was a large study from the U.S. in cancer screening population (25-30). In the 30-39-year-old group, the prevalence of negative high-risk HPV with ASC-US+ (ASC-US or worse) in our study $(1.15 \%: 333 / 29,015)$ was statistically lower than that in the ATHENA study (4.59\%: 562/12,248). (Data 
Table IV. The prevalence of carcinomas by age group in the women with hrHPV-/æL-SIL.

\begin{tabular}{lcccc}
\hline Age & Cervical Ca or adenoca & Endometrial Ca & Total number & Prevalence $(\%)(95 \%$ CI $)$ \\
\hline $20-49$ & 1 & 1 & 71,381 & $0.0028(-0.0026-0.0082)^{\mathrm{a}}$ \\
$50-$ & 1 & 8 & 43,892 & $0.0205(0.0058-0.0353)$ \\
Total & 2 & 9 & 115,273 & $0.0095(0.0034-0.0157)$ \\
\hline
\end{tabular}

${ }^{\text {a }} \mathrm{P}<0.01$ vs. 50-. HPV, human papillomavirus; CI, confidence interval; L-SIL, low grade-squamous intraepithelial lesion; cervical ca or adenoca, cervical cancer or cervical adenocarcinoma; endometrial $\mathrm{Ca}$, endometrial adenocarcinoma.

not shown) (27). It is necessary to consider the possibility that the difference in the prevalence was due to differences in the distribution by age group. Our results suggested the possibility that the prevalence of hrHPV-/ $\geqq$ LSIL in Japan was extremely lower than that in the U.K. or U.S.

Before we introduce the primary HPV testing in Japan, we need to estimate the proportion of women with hrHPV-/ $\geqq$ LSIL /CIN2+. The prevalence of CIN2, CIN3, SCC or cervical adenocarcinoma in all women was $0.03 \%(39 / 115,273)$ in this study and $0.10 \%(25 / 24510)$ in the ARTISTIC study. The prevalence in Japan was significantly lower than that in the U.K.. The prevalence of CIN2, CIN3, SCC or cervical adenocarcinoma in women with $\mathrm{hrHPV}-/ \geqq$ LSIL was $15.3 \%$ $(39 / 255)$ in this study and $8.0 \%(25 / 311)$ in the ARTISTIC study (23). The prevalence in Japan was the same as that in the U.K. $(\mathrm{P}=0.073)$. Therefore, our data suggest that primary HPV testing may have the same efficacy in both the U.K. and Japan.

We were concerned with missed hrHPV negative cervical adenocarcinoma by introducing the primary HPV testing because cervical adenocarcinomas increase and has more hrHPV negative cancer than SCC. Cervical adenocarcinomas account for $15-20 \%$ of cervical cancers (31). Cervical adenocarcinoma can be divided into nine histological groups (17). The highest prevalent histology (75\%) is usual type, and almost all $(80-100 \%)$ are hrHPV+. The secondary high prevalent histology (8\%) is the intestinal type, and almost all (80-100\%) are hrHPV+. Other histology (villoglandular, signet ring cell, endometrioid from the squamous columnar junction zone, serous, clear cell, gastric type, mesonephric) accounted for $7 \%$ or less. Villoglandular, signet ring cell, endometrioid from the squamous columnar junction zone are hrHPV+. Serous and clear cells comprise approximately $30 \%$ of the total. Gastric type and mesonephric are almost all hrHPV negative cancers (17). The prevalence of cervical adenocarcinomas out of the usual type that is missed by the primary HPV testing is quite low. Our data verified that hrHPV negative cervical adenocarcinomas were only one gastric type in cancer screening. We think that concerns over missed cervical adenocarcinoma by primary HPV testing may not be warranted.

The ability to detect endometrial adenocarcinoma has been discovered as an unexpected, major advantage for cervical cancer screening. Although endometrial adenocarcinoma can be missed by primary HPV testing, primary cytology can detect endometrial adenocarcinomas (32). From our data, 3\% of women with hrHPV-/ $\geqq$ LSIL were diagnosed with endometrial adenocarcinomas. Moreover, eight of the nine cases of endometrial adenocarcinoma were discovered in women older than 50 years. Therefore, the prevalence $(0.0205 \%: 9 / 43,892)$ of hrHPV-/ $\geqq$ LSIL carcinomas in women 50 years or older was significantly higher than that $(0.0028 \%$ : $2 / 71,381)$ in women younger than 49 years old. In Japan, the age range of cervical cancer screening is over 20 years-old without the upper limitation. The results of our study showed that the merit in old age group on cervical cancer screening was the discovery for endometrial adenocarcinoma, which wasn't the primary purpose of cervical cancer screening. Therefore, we recommend the cytology for women older than 50 years old. Our data suggest that primary HPV testing had better be introduced in screenings for women younger than 50 years old.

This study has three limitations. First, we did not consider the vaccination rate. Thus, we are concerned that the introduction of primary HPV testing will increase unnecessary colposcopies because the rate of vaccination in Japan is extremely low. Previous reports have verified that the number of colposcopy procedures increased in unvaccinated individuals compared with vaccinated individuals $(33,34)$. In our next study, we will analyse this issue further. Second, HPV testing was not uniform across all regions. The other, we failed to compare the sensitivity and specificity among primary HPV testing, cytology and co-testing because we didn't have the data about hrHPV+/NILM (Negative for intraepithelial lesion or malignancy). However, some manuscripts have indicate the balance between the sensitivity and the specificity in the primary HPV testing is better than the primary cytology $(5,9)$.

In conclusion, hrHPV-/ $\geqq$ LSIL/CIN2+, which can be detected by primary cytology and are missed by primary HPV testing, were clarified in this study and the prevalence was found to be remarkably low in the cancer screening population in Japan. As in other countries using primary HPV testing, it was speculated that the merits were greater than the disadvantages caused by the shift from primary cytology to primary HPV testing. Therefore, our data indicated that primary HPV testing, which was more sensitive for CIN2+ than primary cytology (5), was the feasible method in Japan. Our suggestion was extremely similar with the suggestion in the previous manuscript, which suggested that the fact that there are HPV negative cancers should not undermine all ideas regarding primary HPV screening (17). The difference between primary HPV testing and co-testing in detecting CIN2+ is remarkably small and both of modalities would be effective as a cervical cancer screening program. However, in the setting where medical resource is limited, primary HPV screening should be preferable from the viewpoint of cost-effectiveness. In Japan, Minister of Health, Labour and Welfare announce the guidelines for cervical cancer screening, but actual screening 
program is decided and performed by each municipality. We might recommend to each municipality that primary HPV testing would be chosen according to their financial status. In particular, our result in Table IV showed that women younger than 50 years old had little disadvantage by the shift from primary cytology to primary HPV testing because the number of the women with carcinoma missed by the shift was extremely small. However, our study, which focused on the number of the women with carcinoma missed by the shift, failure to evaluate the lower limit of age range because the number of the women younger than 50 years old, who had carcinoma missed by the shift, was extremely small. Therefore we our result suggested primary HPV testing should be recommended in women younger than 50 years old. A randomized trial should be conducted in the future to compare primary cytology and primary HPV testing and to suggest an adequate algorism and the follow-up system in Japan.

\section{Acknowledgements}

Not applicable.

\section{Funding}

No funding was received.

\section{Availability of data and materials}

The datasets used and/or analyzed during the current study are available from the corresponding author on reasonable request.

\section{Authors' contributions}

TK and MS were responsible for the organization and coordination of the study. TK was the chief investigator and also responsible for the data analysis. YY, OI, TO, TK, MH, HFujit, HFujiw, MY, NS, JK and MS developed the study design. All authors contributed to the writing of the final manuscript. All members of the study contributed to the management or administration of the study. All authors read and approved the final manuscript.

\section{Ethics approval and consent to participate}

The current study was approved by the Ethics Review Board of Fukui University (approval no. 20190094).

\section{Patient consent for publication}

Not applicable.

\section{Competing interests}

The authors declare that they have no competing interests.

\section{References}

1. Ferlay J, Colombet M, Soerjomataram I, Mathers C, Parkin DM, Piñeros M, Znaor A and Bray F: Estimating the global cancer incidence and mortality in 2018: GLOBOCAN sources and methods. Int J Cancer 144: 1941-1953, 2019.
2. Bray F, Ferlay J, Soerjomataram I, Siegel RL, Torre LA and Jemal A: Global cancer statistics 2018: GLOBOCAN estimates of incidence and mortality worldwide for 36 cancers in 185 countries. CA Cancer J Clin 68: 394-424, 2018.

3. Konno R, Konishi H, Sauvaget C, Ohashi Y and Kakizoe T: Effectiveness of HPV vaccination against high grade cervical lesions in Japan. Vaccine 36: 7913-7915, 2018.

4. US Preventive Services Task Force, Curry SJ, Krist AH, Owens DK, Barry MJ, Caughey AB, Davidson KW, Doubeni CA, Epling JW Jr, Kemper AR, et al: Screening for cervical cancer: US preventive services task force recommendation statement. JAMA 320: 674-686, 2018.

5. Ogilvie GS, van Niekerk D, Krajden M, Smith LW, Cook D, Gondara L, Ceballos K, Quinlan D, Lee M, Martin RE, et al: Effect of screening with primary cervical HPV testing vs cytology testing on high-grade cervical intraepithelial neoplasia at 48 months: The HPV FOCAL randomized clinical trial. JAMA 320: 43-52, 2018.

6. Ronco G, Giorgi-Rossi P, Carozzi F, Confortini M, Dalla Palma P, Del Mistro A, Ghiringhello B, Girlando S, Gillio-Tos A, De Marco L, et al: Efficacy of human papillomavirus testing for the detection of invasive cervical cancers and cervical intraepithelial neoplasia: A randomised controlled trial. Lancet Oncol 11: 249-257, 2010.

7. Ronco G, Giorgi-Rossi P, Carozzi F, Dalla Palma P, Del Mistro A, De Marco L, De Lillo M, Naldoni C, Pierotti P, Rizzolo R, et al: Human papillomavirus testing and liquid-based cytology in primary screening of women younger than 35 years: Results at recruitment for a randomised controlled trial. Lancet Oncol 7: 547-555, 2006.

8. Ronco G, Segnan N, Giorgi-Rossi P, Zappa M, Casadei GP, Carozzi F, Dalla Palma P, Del Mistro A, Folicaldi S, Gillio-Tos A, et al: Human papillomavirus testing and liquid-based cytology: Results at recruitment from the new technologies for cervical cancer randomized controlled trial. J Natl Cancer Inst 98: 765-774, 2006.

9. Ronco G, Giorgi-Rossi P, Carozzi F, Confortini M, Dalla Palma P, Del Mistro A, Gillio-Tos A, Minucci D, Naldoni C, Rizzolo R, et al: Results at recruitment from a randomized controlled trial comparing human papillomavirus testing alone with conventional cytology as the primary cervical cancer screening test. J Natl Cancer Inst 100: 492-501, 2008.

10. Ogilvie GS, Krajden M, van Niekerk D, Smith LW, Cook D, Ceballos K, Lee M, Gentile L, Gondara L, Elwood-Martin R, et al: HPV for cervical cancer screening (HPV FOCAL): Complete Round 1 results of a randomized trial comparing HPV-based primary screening to liquid-based cytology for cervical cancer. Int J Cancer 140: 440-448, 2017.

11. Leinonen MK, Nieminen P, Lönnberg S, Malila N, Hakama M, Pokhrel A, Laurila P, Tarkkanen J and Anttila A: Detection rates of precancerous and cancerous cervical lesions within one screening round of primary human papillomavirus DNA testing: Prospective randomised trial in Finland. BMJ 345: e7789, 2012.

12. Canfell K, Caruana M, Gebski V, Darlington-Brown J, Heley S, Brotherton J, Gertig D, Jennett CJ, Farnsworth A, Tan J, et al: Cervical screening with primary HPV testing or cytology in a population of women in which those aged 33 years or younger had previously been offered HPV vaccination: Results of the Compass pilot randomised trial. PLoS Med 14: e1002388, 2017.

13. Solomon D, Davey D, Kurman R, Moriarty A, O'Connor D, Prey M, Raab S, Sherman M, Wilbur D, Wright T Jr, et al: The 2001 Bethesda System: Terminology for reporting results of cervical cytology. JAMA 287: 2114-2119, 2002.

14. KiEY,Lee YK, Lee A and Park JS: Comparison of the PANArray HPV genotyping chip test with the Cobas 4800 HPV and hybrid capture 2 tests for detection of HPV in ASCUS women. Yonsei Med J 59: 662-668, 2018

15. Sauvaget $\mathrm{C}$, Nishino $\mathrm{Y}$, Konno R, Tase $\mathrm{T}$, Morimoto $\mathrm{T}$ and Hisamichi S: Challenges in breast and cervical cancer control in Japan. Lancet Oncol 17: e305-e312, 2016.

16. Kurokawa T, Onuma T, Shinagawa A, Chino Y, Kobayashi M and Yoshida Y: The ideal strategy for cervical cancer screening in Japan: Result from the Fukui cervical cancer screening study. Cytopathology 29: 361-367, 2018.

17. Tjalma W: HPV negative cervical cancers and primary HPV screening. Facts Views Vis Obgyn 10: 107-113, 2018.

18. Clifford GM, Smith JS, Aguado T and Franceschi S: Comparison of HPV type distribution in high-grade cervical lesions and cervical cancer: A meta-analysis. Br J Cancer 89: 101-105, 2003. 
19. Guan P, Howell-Jones R, Li N, Bruni L, de Sanjosé S, Franceschi $\mathrm{S}$ and Clifford GM: Human papillomavirus types in 115,789 HPV-positive women: A meta-analysis from cervical infection to cancer. Int J Cancer 131: 2349-2359: 2012.

20. Onuki M, Matsumoto K, Satoh T, Oki A, Okada S, Minaguchi T, Ochi H, Nakao S, Someya K, Yamada N, et al: Human papillomavirus infections among Japanese women: Age-related prevalence and type-specific risk for cervical cancer. Cancer Sci 100: 1312-1316, 2009

21. Kitchener HC, Almonte M, Wheeler P, Desai M, Gilham C, Bailey A, Sargent A and Peto J; ARTISTIC Trial Study Group: HPV testing in routine cervical screening: Cross sectional data from the ARTISTIC trial. Br J Cancer 95: 56-61, 2006.

22. Kitchener HC, Gilham C, Sargent A, Bailey A, Albrow R, Roberts C, Desai M, Mather J, Turner A, Moss S and Peto J: A comparison of HPV DNA testing and liquid based cytology over three rounds of primary cervical screening: Extended follow up in the ARTISTIC trial. Eur J Cancer 47: 864-871, 2011.

23. Kitchener HC, Almonte M, Thomson C, Wheeler P, Sargent A, Stoykova B, Gilham C, Baysson H, Roberts C, Dowie R, et al: HPV testing in combination with liquid-based cytology in primary cervical screening (ARTISTIC): A randomised controlled trial. Lancet Oncol 10: 672-682, 2009.

24. Sargent A, Bailey A, Almonte M, Turner A, Thomson C, Peto J, Desai M, Mather J, Moss S, Roberts C, et al: Prevalence of type-specific HPV infection by age and grade of cervical cytology: Data from the ARTISTIC trial. Br J Cancer 98: 1704-1709, 2008

25. Castle PE, Stoler MH, Wright TC Jr, Sharma A, Wright TL and Behrens CM: Performance of carcinogenic human papillomavirus (HPV) testing and HPV16 or HPV18 genotyping for cervical cancer screening of women aged 25 years and older: A subanalysis of the ATHENA study. Lancet Oncol 12: 880-890, 2011.

26. Cox JT, Castle PE, Behrens CM, Sharma A, Wright TC Jr and Cuzick J; Athena HPV Study Group: Comparison of cervical cancer screening strategies incorporating different combinations of cytology, HPV testing, and genotyping for HPV 16/18: Results from the ATHENA HPV study. Am J Obstet Gynecol 208: 184. e1-184.e11, 2013.

27. Monsonego J, Cox JT, Behrens C, Sandri M, Franco EL, Yap PS and Huh W: Prevalence of high-risk human papilloma virus genotypes and associated risk of cervical precancerous lesions in a large U.S. screening population: Data from the ATHENA trial. Gynecol Oncol 137: 47-54, 2015.

28. Petry KU, Cox JT, Johnson K, Quint W, Ridder R, Sideri M, Wright TC Jr and Behrens CM: Evaluating HPV-negative CIN2+ in the ATHENA trial. Int J Cancer 138: 2932-2939, 2016.
29. Wright TC Jr, Stoler MH, Behrens CM, Apple R, Derion T and Wright TL: The ATHENA human papillomavirus study: Design, methods, and baseline results. Am J Obstet Gynecol 206: 46.e1-46.e11, 2012

30. Wright TC Jr, Stoler MH, Sharma A, Zhang G, Behrens C and Wright TL; ATHENA (Addressing THE Need for Advanced HPV Diagnostics) Study Group: Evaluation of HPV-16 and HPV-18 genotyping for the triage of women with high-risk HPV+ cytology-negative results. Am J Clin Pathol 136: 578-586, 2011.

31. Young RH and Clement PB: Endocervical adenocarcinoma and its variants: Their morphology and differential diagnosis. Histopathology 41: 185-207, 2002.

32. Schnatz PF, Guile M, O'Sullivan DM and Sorosky JI: Clinical significance of atypical glandular cells on cervical cytology. Obstet Gynecol 107: 701-708, 2006.

33. Lew JB, Simms KT, Smith MA, Hall M, Kang YJ, Xu XM, Caruana M, Velentzis LS, Bessell T, Saville M, et al: Primary HPV testing versus cytology-based cervical screening in women in Australia vaccinated for HPV and unvaccinated: Effectiveness and economic assessment for the national cervical screening program. Lancet Public Health 2: e96-e107, 2017.

34. Canfell K, Saville M, Caruana M, Gebski V, Darlington-Brown J, Brotherton J, Heley S and Castle PE: Protocol for Compass: A randomised controlled trial of primary HPV testing versus cytology screening for cervical cancer in HPV-unvaccinated and vaccinated women aged 25-69 years living in Australia. BMJ Open 8: e016700, 2018.

This work is licensed under a Creative Commons Attribution-NonCommercial-NoDerivatives 4.0 International (CC BY-NC-ND 4.0) License. 